\title{
APRENDER PARA EL FUTURO
}

Aparicio-Gómez, Oscar-Yecid

https://orcid.org/0000-0003-3535-6288

Ostos-Ortiz, Olga-Lucía

https://orcid.org/0000-0002-6477-9872

DOI: $10.13140 / R G .2 .2 .16070 .70726$

\section{Resumen}

Uno de los grandes desafíos de la educación actual consiste en preparar a los estudiantes para el trabajo y para la vida; es decir, es importante pensar en una educación dinámica, acompañada de una correcta evaluación dinámica que sea prospectiva, que se proyecte hacia el futuro, y un futuro impredecible, un futuro incierto en entornos laborales cambiantes. Es importante tener en cuenta, cuando nos ponemos al frente o al lado de un estudiante para acompañarle en su proceso de aprendizaje, que estamos preparándolo para lo que no sabemos y para lo que él tampoco sabe.

Palabras clave: Educación, Educación superior, Educación universitaria, Innovación educacional, Extensión universitaria, Formación a distancia, Teleformación, Educación basada en las competencias, Perfeccionamiento, Tendencia educacional, Educación del mañana, Educación permanente, Aprendizaje continuo, Educación continua, Formación continua, Desarrollo moral, Ética.

\section{¿Cómo citar este working paper?}

Aparicio-Gómez, Oscar-Yecid y Ostos-Ortiz, Olga-Lucía (2020). Aprender para el futuro. Universidad Santo Tomás. Working Paper No. 197565

\section{Aprender para el futuro}

Estamos preparando a los estudiantes para la incertidumbre. Las universidades se enfrentan estos desafíos continuos de conectar el aprendizaje formal con los problemas del mundo real, y la gran pregunta que se nos plantea ahora es cómo pueden las personas comprender el aprendizaje en 
términos de disposición para aprender no solamente la adquisición de conceptos, sino especialmente la profundización de conocimientos. Este cambio requiere pedagogías que se centren en la obtención de habilidades para aprender y aprender a aprender, y que supone una disposición para cambiar de perspectiva, escuchar respetuosamente el punto de vista de otro.

Los datos a los que accedemos cada vez son distintos, y la manera como se comprenden también lo son; por lo tanto, el acceso a la información, así como la comprensión e interpretación de los datos, reclaman competencias adecuadas para mantener activo ese aprendizaje a lo largo de la vida. En este caso debemos pensar en el enfoque de aprendizaje apropiado para que las personas puedan aprender continuamente a ser ciudadanos responsables

Se trata, por lo tanto, de abrir posibilidades y perspectivas para agenciar la autonomía, la propia autonomía, para planificar qué y cómo aprender, para que se tengan los criterios necesarios para adaptar las habilidades cognitivas y los conocimientos, apropiándose el uso de la tecnología para el propio aprendizaje.

\section{Conclusiones}

El aprendizaje para apropiarse del propio futuro, conforme éste va haciendo el arribo a nuestro momento histórico, requiere que esté muy bien situado, en términos adaptativos, pero también desde el pensamiento crítico, impulsados por la creatividad y la innovación como referentes. Se trata de reconocer en qué términos podríamos hablar nosotros en el contexto digital, en los entornos digitales,

y cómo acceder a aprendizajes personalizados, actualizados por analíticas de aprendizaje, para que la adaptación de los procesos de aprendizaje pueda contextualizarse y recontextualizarse más allá del aula de clase presencial o virtual.

\section{Referencias}

Aparicio-Gómez, Oscar-Yecid (2006). El nexo familia - persona en la filosofía personalista. Universidad de Barcelona. ISBN: 846-899-781-1.

Aparicio-Gómez, Oscar-Yecid (2016). La persona en la Sociedad Digital. En Aparicio-Gómez, Oscar-Yecid (Eds.). El Uso Educativo de las TIC (pp. 251-256). Bogotá, Colombia: Universidad Central. ISBN: 978-958-26-0316-8

Aparicio-Gómez, Oscar-Yecid (2017). Uso de las TIC en el Colegio Padre Manyanet - Chía, 
Colombia (2011-2014). En Aparicio-Gómez, Oscar-Yecid y Aparicio-Gómez, William-Oswaldo (Eds.). Uso de las TIC para la innovación (pp. 129-158). Bogotá, Colombia: Ed\&TIC. ISBN: 978958-26-0367-0.

Aparicio-Gómez, Oscar-Yecid (2018). La investigación escolar. En: Revista Interamericana de Investigación, Educación y Pedagogía, RIIEP. Vol. 11, Núm. 2. p. 121 - 133. ISSN: 1657-107X - eISSN: 2500-5421. DOI: https://doi.org/10.15332/s1657-107X.2018.0002.08

Aparicio-Gómez, Oscar-Yecid (2018). Las TIC como herramientas cognitivas. En: Revista Interamericana de Investigación, Educación y Pedagogía, RIIEP. Vol. 11, Núm. 1. p. 67 - 80. ISSN: 1657-107X - e-ISSN: 2500-5421. DOI: https://doi.org/10.15332/s1657-107X.2018.0001.07

Aparicio-Gómez, Oscar-Yecid (2019). El uso educativo de las TIC. En: Revista Interamericana de Investigación, Educación y Pedagogía, RIIEP. Vol. 12, Núm. 1. p. 211 - 227. ISSN: 1657-107X - eISSN: 2500-5421. DOI: https://doi.org/10.15332/s1657-107X.2019.0001.02

Aparicio-Gómez, Oscar-Yecid (2019). Uso y apropiación de las TIC en educación. En: Revista Interamericana de Investigación, Educación y Pedagogía, RIIEP. Vol. 12, Núm. 1. p. 253 - 284. ISSN: 1657-107X - e-ISSN: 2500-5421. DOI: https://doi.org/10.15332/s1657-107X.2019.0001.04

Aparicio-Gómez, Oscar-Yecid (2020). El oficio de sabio: Indagar, investigar, innovar. En: AparicioGómez, Oscar-Yecid y Ostos-Ortiz, Olga-Lucía. (Eds.). Innovación Educativa y Gestión del Conocimiento (pp. 177-192). Bogotá, Colombia: Universidad Santo Tomás. ISBN: 978-958-782$304-2$

Aparicio-Gómez, Oscar-Yecid (2020). The education of desire and the use of ICT. In Bosch, M. (Ed.). Desire and Human Flourishing: Perspectives from Positive Psychology, Moral education and Virtue Ethics. New York, USA: Springer Publishing. ISBN: 978-3-030-47001-2

Aparicio-Gómez, Oscar-Yecid (Ed.) (2016). El Uso Educativo de las TIC. Bogotá, Colombia: Universidad Central. ISBN: 978-958-26-0316-8.

Aparicio-Gómez, Oscar-Yecid y Aparicio-Gómez, William-Oswaldo. (Eds.) (2017). Uso de las TIC para la innovación. Bogotá, Colombia: Ed\&TIC. ISBN: 978-958-56300-0-0.

Aparicio-Gómez, Oscar-Yecid y Ostos-Ortiz, Olga-Lucía (2020). Aprender en tiempos de incertidumbre. Universidad Santo Tomás. Working Paper No. 197506. DOI: 10.13140/RG.2.2.11467.21284

Aparicio-Gómez, Oscar-Yecid y Ostos-Ortiz, Olga-Lucía (2020). Aprender con tecnología. Universidad Santo Tomás. Working Paper No. 197508. DOI: 10.13140/RG.2.2.10418.63689

Aparicio-Gómez, Oscar-Yecid y Ostos-Ortiz, Olga-Lucía (2020). Aprender con recursos educativos abiertos. Universidad Santo Tomás. Working Paper No. 197509. DOI: 10.13140/RG.2.2.30551.29605 
Aparicio-Gómez, Oscar-Yecid y Ostos-Ortiz, Olga-Lucía (2020). Aprender con Big Data. Universidad Santo Tomás. Working Paper No. 197510. DOI: 10.13140/RG.2.2.33906.73924

Aparicio-Gómez, Oscar-Yecid y Ostos-Ortiz, Olga-Lucía (2020). Aprendizaje continuo. Universidad Santo Tomás. Working Paper No. 197511. DOI: 10.13140/RG.2.2.14612.94081

Aparicio-Gómez, Oscar-Yecid y Ostos-Ortiz, Olga-Lucía (2020). Aprendizaje en red sin conexión. Universidad Santo Tomás. Working Paper No. 197512. DOI: 10.13140/RG.2.2.33067.87840cre Aparicio-Gómez, Oscar-Yecid y Ostos-Ortiz, Olga-Lucía (2020). Design thinking para resolver problemas. Universidad Santo Tomás. Working Paper No. 197514. DOI: 10.13140/RG.2.2.10838.06721

Aparicio-Gómez, Oscar-Yecid y Ostos-Ortiz, Olga-Lucía (2020). Flipped learning para el acompañamiento y la independencia. Universidad Santo Tomás. Working Paper No. 197516. DOI: 10.13140/RG.2.2.17845.09444

Aparicio-Gómez, Oscar-Yecid y Ostos-Ortiz, Olga-Lucía (2020). Gamification para aprender a través de juegos. Universidad Santo Tomás. Working Paper No. 197517. DOI: 10.13140/RG.2.2.14489.65126

Aparicio-Gómez, Oscar-Yecid y Ostos-Ortiz, Olga-Lucía (2020). Investigación, innovación y transferencia del conocimiento. Universidad Santo Tomás. Working Paper No. 197503. Universidad Santo Tomás. Working Paper No. 197503. DOI: 10.13140/RG.2.2.17488.33282

Aparicio-Gómez, Oscar-Yecid y Ostos-Ortiz, Olga-Lucía (2020). Metodologías activas para el aprendizaje. Universidad Santo Tomás. Working Paper No. 197513. DOI: 10.13140/RG.2.2.29712.43521

Aparicio-Gómez, Oscar-Yecid y Ostos-Ortiz, Olga-Lucía (2020). Pedagogías emergentes. Universidad Santo Tomás. Working Paper No. 197507. DOI: 10.13140/RG.2.2.26566.70725

Aparicio-Gómez, Oscar-Yecid y Ostos-Ortiz, Olga-Lucía (2020). Social media para el aprendizaje en red. Universidad Santo Tomás. Working Paper No. 197518. DOI: 10.13140/RG.2.2.21200.53760 Aparicio-Gómez, Oscar-Yecid y Ostos-Ortiz, Olga-Lucía (2020). Teachback para aprender a través de la conversación. Universidad Santo Tomás. Working Paper No. 197515. DOI: 10.13140/RG.2.2.17548.95368

Aparicio-Gómez, Oscar-Yecid y Ostos-Ortiz, Olga-Lucía (Eds.) (2020). Innovación Educativa y Gestión del Conocimiento. Bogotá, Colombia: Universidad Santo Tomás. ISBN: 978-958-782-3042 177-192

Aparicio-Gómez, Oscar-Yecid y Venegas, D. (2016). Proyecto Ágora: Un espacio virtual para afianzar el aprendizaje escolar y brindar herramientas para la construcción de nuevos conocimientos. En Aparicio-Gómez, Oscar-Yecid (Ed.), El Uso Educativo de las TIC (pp. 115-124). Bogotá, 
Colombia: Universidad Central. ISBN: 978-958-26-0316-8

Aparicio-Gómez, Oscar--Yecid, Abadía, C. (2019). La investigación en la educación. En: Revista Interamericana de Investigación, Educación y Pedagogía, RIIEP. Vol. 12, Núm. 1. p. 229 - 251. ISSN: 1657-107X - e-ISSN: 2500-5421. DOI: https://doi.org/10.15332/s1657-107X.2019.0001.03

Aparicio-Gómez, Oscar-Yecid, Ostos-Ortiz, Olga-Lucía (2018). El constructivismo y el construccionismo. En: Revista Interamericana de Investigación, Educación y Pedagogía, RIIEP. Vol. 11, Núm. 2. p. 115 - 120. ISSN: 1657-107X - e-ISSN: 2500-5421. DOI: https://doi.org/10.15332/s1657-107X.2018.0002.05

Aparicio-Gómez, Oscar-Yecid, Ostos-Ortiz, Olga-Lucía (2018). Las TIC como herramientas cognitivas para la investigación. En: Revista Interamericana de Investigación, Educación y Pedagogía, RIIEP. Vol. 11, Núm. 1. p. 81 - 86. ISSN: 1657-107X - e-ISSN: 2500-5421. DOI: https://doi.org/10.15332/s1657-107X.2018.0001.08

Aparicio-Gómez, Oscar-Yecid, Ostos-Ortiz, Olga-Lucía, Cortés, M. (2019). Redes sociales, tejidos de paz. En: Revista Hallazgos. Vol. 16, Núm. 32. p. 17 - 25. ISSN: 1794-3841 - e-ISSN: 2422-409X - DOI: https://doi.org/ 10.15332/s1794-3841

Aparicio-Gómez, Oscar-Yecid, Ostos-Ortiz, Olga-Lucía, Cortés, M., Abadía, D. (2019). Análisis sistemático de los artículos publicados en la revista interamericana de educación, investigación y pedagogía (RIIEP) durante 2014 a 2019. En: Revista Interamericana de Investigación, Educación y Pedagogía, RIIEP. Vol. 12, Núm. 2. p. 225 - 240. ISSN: 1657-107X - e-ISSN: 2500-5421. DOI: https://doi.org/10.15332/s1657107X.2019.0001.02

Aparicio-Gómez, William-Oswaldo y Aparicio-Gómez, Oscar-Yecid (2017). Investigación y uso de las TIC: La innovación en el contexto educativo colombiano. En O. Aparicio-Gómez, Oscar-Yecid y Aparicio-Gómez, William-Oswaldo (Eds.). Uso de las TIC para la innovación (pp. 17-36). Bogotá, Colombia: Ed\&TIC. ISBN: 978-958-26-0367-0.

Gonzáles-David, Johana-Lizeth; Ostos-Ortíz, Olga-Lucía. y Aparicio-Gómez, Oscar-Yecid. (2020). Convocatoria de Medición de Revistas Científicas. Universidad Santo Tomás. Working Paper No 197546. DOI: 10.13140/RG.2.2.25036.44165

Herrera, M., Aparicio-Gómez, Oscar-Yecid y otros (2017). Ecoturismo sostenible apoyado en las TIC en el ecosistema de alta montaña "La Montaña del Oso", Vereda Yerbabuena (Chía - Cundinamarca). En Aparicio-Gómez, Oscar-Yecid y Aparicio-Gómez, William-Oswaldo (Ed.). Uso de las TIC para la innovación (pp. 159-179). Bogotá, Colombia: Ed\&TIC. ISBN: 978-958-26-0367-0.

Niemi, H., Multisilta, J., Lipponen, L. \& Vivitsou, M. (eds.) (2014). Finnish Innovations and Technologies in Schools: A Guide towards New Ecosystems of Learning. Rotterdam: Sense Publishers. 
Ostos-Ortíz, Olga-Lucía (2020). Bases del Sistema Institucional de Investigación. Universidad Santo Tomás. Working Paper No 197528. DOI: 10.13140/RG.2.2.17486.69447

Ostos-Ortíz, Olga-Lucía (2020). Campos de acción USTA. Universidad Santo Tomás. Working Paper No 197529. DOI: 10.13140/RG.2.2.14131.25126

Ostos-Ortíz, Olga-Lucía (2020). Convocatoria Semilleros 2017. Universidad Santo Tomás. Working Paper No 197530. DOI: 10.13140/RG.2.2.24852.94084

Ostos-Ortíz, Olga-Lucía (2020). Cooperación Interinstitucional para el fortalecimiento de la calidad editorial, científica, la visibilidad y el impacto en publicación científica seriada. Universidad Santo Tomás. Working Paper No 197560. DOI: 10.13140/RG.2.2.17086.92481

Ostos-Ortíz, Olga-Lucía (2020). Documento marco de investigación, innovación, creación artística cultural. Universidad Santo Tomás. Working Paper No 197532. DOI: 10.13140/RG.2.2.20842.13763 Ostos-Ortíz, Olga-Lucía (2020). Gestión de la Investigación Catálogo de Proyectos 2017. Universidad Santo Tomás. Working Paper No 197534. DOI: 10.13140/RG.2.2.33864.14089

Ostos-Ortíz, Olga-Lucía (2020). Gestión de la Investigación Catálogo de Proyectos 2018. Universidad Santo Tomás. Working Paper No 197535. DOI: 10.13140/RG.2.2.12053.76002

Ostos-Ortíz, Olga-Lucía (2020). Gestión de la Investigación CP2010-14. Universidad Santo Tomás. Working Paper No 197536. DOI: 10.13140/RG.2.2.25475.53285

Ostos-Ortíz, Olga-Lucía (2020). Gestión de la Investigación FODEIN2017. Universidad Santo Tomás. Working Paper No 197540. DOI: 10.13140/RG.2.2.26425.80487

Ostos-Ortíz, Olga-Lucía (2020). Gestión de la Investigación FODEIN2018. Universidad Santo Tomás. Working Paper No 197541. DOI: 10.13140/RG.2.2.25849.08802

Ostos-Ortíz, Olga-Lucía (2020). Gestión de la Investigación FODEIN2021. Universidad Santo Tomás. Working Paper No 197542. DOI: 10.13140/RG.2.2.19138.20165

Ostos-Ortíz, Olga-Lucía (2020). Gestión de la Investigación Medición2019. Universidad Santo Tomás. Working Paper No 197543. DOI: 10.13140/RG.2.2.12453.52969

Ostos-Ortíz, Olga-Lucía (2020). Gestión de la Investigación Perfiles Especializados de Investigación - División de Ciencias Económicas USTA. Universidad Santo Tomás. Working Paper No 197545. DOI: $10.13140 /$ RG.2.2.30430.05443

Ostos-Ortíz, Olga-Lucía (2020). Gestión de la Investigación Plan Estratégico 2017. Universidad Santo Tomás. Working Paper No 197539. DOI: 10.13140/RG.2.2.36492.13440

Ostos-Ortíz, Olga-Lucía (2020). Gestión de la Investigación Plan Estratégico de la Investigación USTA2018. Universidad Santo Tomás. Working Paper No 197547. DOI: 10.13140/RG.2.2.21680.99849

Ostos-Ortíz, Olga-Lucía (2020). Gestión de la Investigación USTA2015. Universidad Santo Tomás. 
Working Paper No 197537. DOI: 10.13140/RG.2.2.15409.20320

Ostos-Ortíz, Olga-Lucía (2020). Gestión de la Investigación USTA2017. Universidad Santo Tomás. Working Paper No 197538. DOI: 10.13140/RG.2.2.28830.97603

Ostos-Ortíz, Olga-Lucía (2020). Gobernanza Universitaria y Gestión de la Investigación 2017. Universidad Santo Tomás. Working Paper No 197549. DOI: 10.13140/RG.2.2.20003.27688

Ostos-Ortíz, Olga-Lucía (2020). Producción Editorial Universidad a Distancia. Universidad Santo Tomás. Working Paper No 197554. DOI: 10.13140/RG.2.2.31557.27367

Ostos-Ortíz, Olga-Lucía (2020). Redes de Investigación USTA. Universidad Santo Tomás. Working Paper No 197556. DOI: 10.13140/RG.2.2.34912.71685

Ostos-Ortiz, Olga-Lucía y Aparicio-Gómez, Oscar-Yecid (2020). ¿Qué es visibilidad e impacto de la producción académica? Universidad Santo Tomás. Working Paper No. 197502. DOI: 10.13140/RG.2.2.26991.05284

Ostos-Ortiz, Olga-Lucía y Aparicio-Gómez, Oscar-Yecid (2020). Análisis del conflicto. Universidad Santo Tomás. Working Paper No. 197501. DOI: 10.13140/RG.2.2.13569.28002

Ostos-Ortíz, Olga-Lucía. y Aparicio-Gómez, Oscar-Yecid. (2020). ¿Cómo identificar el tejido de la producción científica de las instituciones y países?: Métricas alternativas. Universidad Santo Tomás. Working Paper No 197557. DOI: 10.13140/RG.2.2.29879.55209

Ostos-Ortíz, Olga-Lucía. y Aparicio-Gómez, Oscar-Yecid. (2020). ¿Qué es y cómo funciona Scielo? Universidad Santo Tomás. Working Paper No 197561. DOI: 0.13140/RG.2.2.30508.69762

Ostos-Ortíz, Olga-Lucía. y Aparicio-Gómez, Oscar-Yecid. (2020). Autores, evaluadores y bases bibliográficas: el rol del editor. Universidad Santo Tomás. Working Paper No 197558. DOI: 10.13140/RG.2.2.23168.66563

Ostos-Ortíz, Olga-Lucía. y Aparicio-Gómez, Oscar-Yecid. (2020). Bases Biológicas de la creatividad. Universidad Santo Tomás. Working Paper No 197526. DOI: 10.13140/RG.2.2.22939.28966

Ostos-Ortíz, Olga-Lucía. y Aparicio-Gómez, Oscar-Yecid. (2020). Bases científicas de la intuición. Universidad Santo Tomás. Working Paper No 197527. DOI: 10.13140/RG.2.2.10775.80800

Ostos-Ortíz, Olga-Lucía. y Aparicio-Gómez, Oscar-Yecid. (2020). Bases Biológicas de la creatividad. Universidad Santo Tomás. Working Paper No 197551. DOI:

Ostos-Ortíz, Olga-Lucía. y Aparicio-Gómez, Oscar-Yecid. (2020). Diseño y Gestión de Proyectos de Investigación. Universidad Santo Tomás. Working Paper No 197531. DOI: 10.13140/RG.2.2.14786.61120

Ostos-Ortíz, Olga-Lucía. y Aparicio-Gómez, Oscar-Yecid. (2020). Escritura científica. Universidad Santo Tomás. Working Paper No 197533. DOI: 10.13140/RG.2.2.20442.36807 
Ostos-Ortíz, Olga-Lucía. y Aparicio-Gómez, Oscar-Yecid. (2020). Interdisciplinariedad. Universidad Santo Tomás. Working Paper No 197548. DOI: 10.13140/RG.2.2.26714.16324

Ostos-Ortíz, Olga-Lucía. y Aparicio-Gómez, Oscar-Yecid. (2020). ORCID: Identificación Universal de Autores. Universidad Santo Tomás. Working Paper No 197553. DOI: 10.13140/RG.2.2.11424.61449

Ostos-Ortíz, Olga-Lucía. y Aparicio-Gómez, Oscar-Yecid. (2020). Redes científicas, una aproximación biológica. Universidad Santo Tomás. Working Paper No 197555. DOI: 10.13140/RG.2.2.14780.05768

Ostos-Ortíz, Olga-Lucía. y Aparicio-Gómez, Oscar-Yecid. (2020). Usos de Latindex. Universidad Santo Tomás. Working Paper No 197550. DOI: 10.13140/RG.2.2.16647.83362

Ostos-Ortíz, Olga-Lucía. y Aparicio-Gómez, Oscar-Yecid. (2020). Uso e interpretación de las métricas científicas en el sector editorial. Universidad Santo Tomás. Working Paper No 197552. DOI: 10.13140/RG.2.2.35751.57760

Quintana J., Aparicio-Gómez, Oscar-Yecid (Eds.) (2017). Temas emergentes en educación. Bogotá, Colombia: Universidad Central. ISBN: 978-958-26-0367-0.

Rodríguez, M. V. y Aparicio-Gómez, Oscar-Yecid (2017). Uso y apropiación de las TIC como herramientas cognitivas para el aprendizaje en el Departamento de Contaduría Pública de la Universidad Central. En Aparicio-Gómez, Oscar-Yecid y Aparicio-Gómez, William-Oswaldo (Eds.). Uso de las TIC para la innovación (pp. 37-46). Bogotá, Colombia: Ed\&TIC. ISBN: 978-958-26-03670.

Sheninger, E. (2015). Leading Future-Ready Schools. International Center for Leadership in Education, Rexford, USA

Venegas, D. y Aparicio-Gómez, Oscar-Yecid (2016). Ambientes virtuales de aprendizaje para la implementación del sistema integrado de gestión como estrategia para el mejoramiento del aprendizaje. En Aparicio-Gómez, Oscar-Yecid (Ed.), El Uso Educativo de las TIC (pp. 233-250). Bogotá, Colombia: Universidad Central. ISBN: 978-958-26-0316-8 